\title{
Impact of a temporal sinusoidal phase modulation on the optical spectrum
}

\author{
C Finot, ${ }^{1}$ F Chaussard, ${ }^{1}$ and S Boscolo ${ }^{2}$ \\ ${ }^{1}$ Laboratoire Interdisciplinaire Carnot de Bourgogne, UMR 6303 CNRS - Université de \\ Bourgogne-Franche-Comté, 9 avenue Alain Savary, BP 47870, 21078 Dijon Cedex, France \\ ${ }^{2}$ Aston Institute of Photonic Technologies, School of Engineering and Applied Science, Aston \\ University, Birmingham B4 7ET, United Kingdom \\ E-mail : christophe.finot@u-bourgogne.fr
}

\begin{abstract}
We discuss the effects of imparting a temporal sinusoidal phase modulation to a continuous wave on the frequency spectrum. While a practical analytical solution to this problem already exists, we present here a physical interpretation based on interference processes. This simple model will help the students better understand the origin of the oscillatory structure that can be observed in the resulting spectrum and that is characteristic of Bessel functions of the first kind. We illustrate our approach with an example from the field of optics.
\end{abstract}

Keywords. Phase modulation, Fourier analysis, Bessel functions of the first kind.

\section{Introduction}

Transmission of an information-bearing signal requires the modulation of a continuous wave. One of the most widely used families of continuous-wave modulation systems is amplitude modulation, in which the amplitude of a periodic waveform, called the carrier signal, is varied in proportion to that of the message signal being transmitted. A second family of continuous-wave modulation systems is angle modulation, which involves variation of the angle (instantaneous phase) of the carrier wave with the varying amplitude of the message signal. The family comprises frequency modulation, in which the message signal causes a functional variation of the carrier frequency, and phase modulation, in which the instantaneous phase deviation is controlled by the modulating waveform, such that the principle frequency remains constant. Angle modulation forms the basis for the worldwide used broadcasting of radio programming using frequency-modulation technology or the coherent optical communications [1].

In this paper, we discuss the simplest form of phase modulation in which the modulating signal is a sinusoid. The waveform of a signal and its spectrum (i.e., frequency content) are two natural vehicles to understand the signal. Following the work of Joseph Fourier [2] who was born in Burgundy, France 250 years ago, whenever an operation is performed on the waveform of a signal in the time domain (real space), a corresponding 
modification is applied to the spectrum of the signal in the frequency domain (reciprocal space), and vice versa. Therefore, here we want to form an idea of the corollary that the application of a phase modulation in the time domain has in the frequency domain. Rather than relying on Fourier transformations involving special functions, we base our approach on the physical interpretation of a phase modulation and basic mathematical tools that any physics or engineering undergraduate student should be familiar with. To illustrate our approach, we use the example of an optical wave.

After introducing the problem being studied, we describe our proposed approach and how the resulting evaluation of different phase contributions leads to a fairly accurate description of the spectral envelope of the modulated wave. We then describe further approximations that can be used for the central and outermost parts of the spectrum and that provide some analytical insights into the influence of various relevant parameters on the spectrum.

\section{Problem under investigation}

The problem being investigated is rather simple. We consider a phase-modulated sinusoidal wave, which can be represented in complex exponential form by the equation

$$
E(t)=\psi(t) e^{i \omega_{p} t}=\psi_{0} e^{i\left[\omega_{p} t+\varphi(t)\right]}
$$

where $\psi_{0}$ is the constant amplitude of the wave, the exponential-term is the carrier at its angular frequency $\omega_{p}$ and the instantaneous phase deviation $\varphi(t)$, and $\psi(t)$ is the slowly-varying wave envelope. We assume the most basic periodic modulation, i.e. a sinusoidal pattern [Fig. 1(a)], which reads:

$$
\varphi(t)=A \cos \left(\omega_{c} t\right)
$$

where $A$ is the amplitude of the modulation and $\omega_{c}$ is its angular frequency.

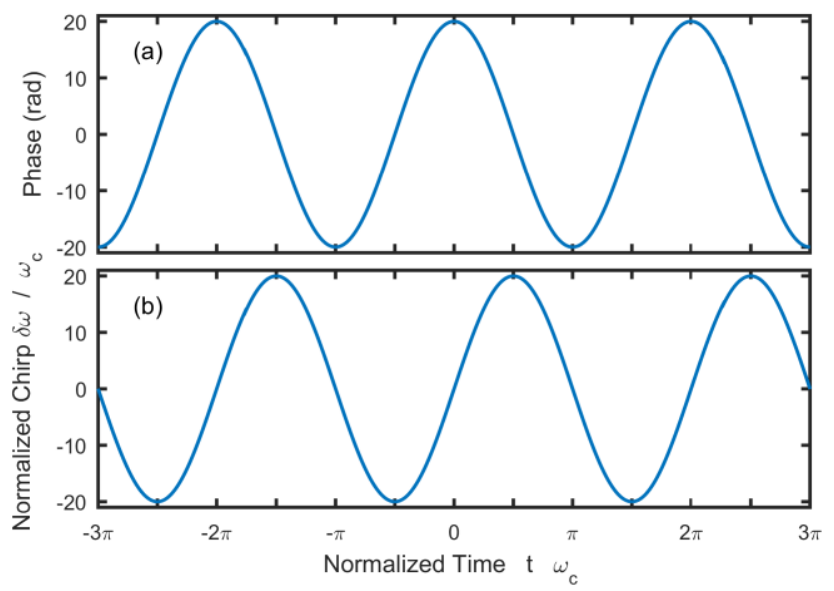

Figure 1 - (a) Sinusoidal temporal phase modulation $\varphi(t)$ with amplitude $A=20 \mathrm{rad}$. (b) Corresponding chirp $\delta \omega(t)$.

Our goal is to elucidate the effects of this phase modulation on the spectrum of frequency components of the wave envelope by means of physical considerations. The spectrum of $\psi(t)$ is given by its Fourier transform:

$$
s(\omega)=\int_{-\infty}^{\infty} \psi(t) e^{i \omega t} d t \propto \int_{-\infty}^{\infty} e^{i A \cos \left(\omega_{c} t\right)} e^{i \omega t} d t
$$

In the absence of any modulation, $s(\omega)$ would be a Dirac delta function centered at $\omega=0$. If only the amplitude of the envelope $\psi_{0}$ was modulated by a sinusoidal wave, the resulting spectrum would consist of two Diracpeaks located at the positive and negative carrier frequency $\omega_{c}$. In the case of a sinusoidal modulation of the 
phase, the spectrum of $\psi(t)$ can be obtained by expanding $\psi(t)$ in series of Bessel functions of the first kind and integer order. These are special mathematical functions denoted by $J_{n}(x)$, where $n$ represents the order of the function [3,4]. Bessel functions appear in many different problems of wave propagation and static potentials, ranging from the study of the far-field diffraction through a circular aperture [5] to the vibration of a cantilever beam or a circular membrane [6]. They are today implemented in most of the scientific computation and programming software. The general trends of the Bessel functions of the first kind and integer order are illustrated in Fig. 2. We can notice that, as a function of $x$, all the curves are damped oscillatory. Except for $J_{0}(x)$, they start from zero, rise to a positive maximum, then oscillate about zero with ever decreasing amplitudes. The exception, $J_{0}(x)$, starts from an amplitude of unity. The graphs look roughly like oscillating sine or cosine functions that decay proportionally to $1 / \sqrt{x}$, although their roots are not generally periodic, except asymptotically for large $x$. One would also need to know that $J_{-n}(x)=(-1)^{n} J_{n}(x)$. By a careful examination of the resulting, rather complex equation describing the spectrum of $\psi(t)$ (not given here) and the plots of the Bessel functions in Fig. 2, one could deduce the following properties. The spectrum consists of a discrete set of spectral lines located at $\omega= \pm n \omega_{c}$ from the central spectral component (number $n=0$ ), hence spaced $\omega_{c} \mathrm{rad} / \mathrm{s}$ apart. The amplitude of the $\pm n^{\text {th }}$ spectral line from the centre is

$$
s_{n} \propto J_{n}(A)
$$

thus, the spectrum is symmetric about the central component. While the width of the spectrum is mathematically infinite $\left(J_{n}(A)\right.$ only approaches 0 as $n$ approaches infinity), in physics terms the signal is considered confined within limits which contain all significant components. As the amplitude of the modulation $A$ increases, the number of significant spectral components increases, hence the spectrum broadens. The angular frequency of the modulation $\omega_{c}$ affects the extent of the spectrum through the size of the line spacing.
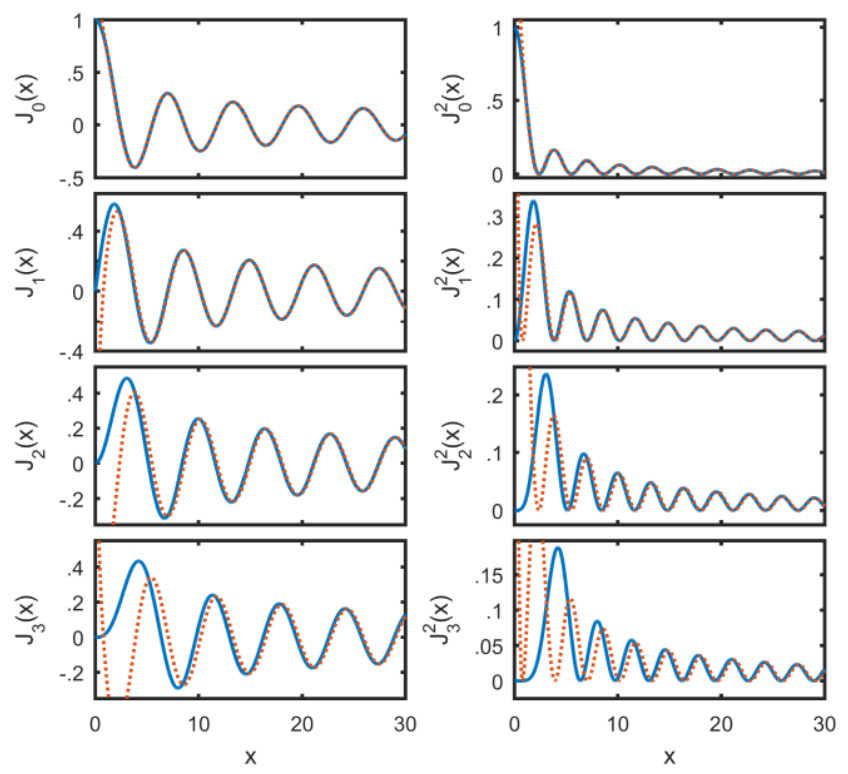

Figure 2 - Plots of Bessel functions of the first kind and integer order $J_{n}(x)$ and their squares (solid blue curves). The dashed red curves represent the asymptotic forms of the functions for large arguments as given by Eqs. (30) and (31).

The main issue with this conventional approach to the analysis of the spectrum of a phase-modulated signal is that the student should commit to memory the general trends of the Bessel functions. In the next sections, we will provide a physical explanation for various general features of a phase-modulated signal's spectrum, which 
should ease the student's understanding of the nature of this spectrum. We will develop a time-domain model based on interference processes, which physics students discover in courses on waves [7, 8]. As the wave interference phenomenon is usually introduced through the single- or double-slit experiments in optics [5], we will base our discussion on the case of an optical wave. In this case, modulations with frequencies of tens of gigahertz and amplitudes of several tens of radians are feasible with current electro-optic phase modulators [9] or through cross-phase modulation [10]. The use of a sinusoidal phase modulation of an optical signal has found numerous applications in devices aimed at generating ultrashort pulse trains [11] or achieving ultrafast processing such as temporal magnification [9] and optical sampling [12]. For optical waves, the most relevant quantity that can be assessed through experiments is not the spectrum $s(\omega)$ but its absolute square $S(\omega)=|s(\omega)|^{2}$, which can be directly recorded with an optical spectrum analyzer. Therefore, we can deduce from Eq. (4) that the optical spectrum will consist of spectral lines of amplitudes $S_{n} \propto J_{n}^{2}(A)$. This entails twice faster oscillations and higher rate of decay of the spectral lines as a function of $A$ [see Fig. 2(b)].

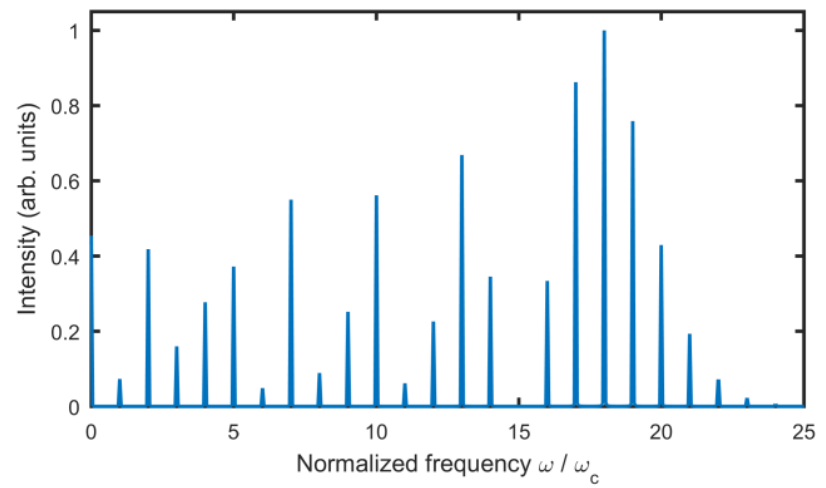

Figure 3 - Optical spectrum $S_{n}$ obtained for a phase modulation with amplitude $A=20 \mathrm{rad}$ (only the positive frequencies are plotted).

One of the crucial ingredients of our approach is the chirp of an optical signal, denoted here by $\delta \omega(t)$, and defined as the opposite of the first derivative with respect to time of the instantaneous phase deviation: $\delta \omega(t)=-d \varphi / d t$ [Fig. 1(b)]. It is usually understood as the time dependence of the instantaneous frequency of the signal, the instantaneous frequency being the time derivative of the instantaneous phase $\omega_{p} t+\varphi(t)$. It is worth noting here that mathematically speaking the two forms of angle modulation used in communication systems are almost identical twins. The only difference is that frequency modulation corresponds to a modulation pattern which is the differential of that produced by phase modulation. In the following section, we discuss how the spectrum $S(\omega)$ can be inferred from the knowledge of the chirp $\delta \omega(t)$.

\section{Wave interference model}

\subsection{Estimation of the spectral width}

The chirp corresponding to the phase modulation being studied is given by

$$
\delta \omega(t)=A \omega_{c} \sin \left(\omega_{c} t\right)
$$

Hence, the instantaneous frequency of the signal oscillates at the same frequency as the phase modulation. Given the evenness of the phase modulation function and oddness of the chirp function, we can restrict our study to the positive times only. In the first instance, we will consider a single half-period of oscillation, between $t=0$ and $t=\pi / \omega_{c}$ (Fig. 4). The chirp is null at $t=0$ and has a maximum value of $\omega_{m}=A \omega_{c}$ at $t_{m}=$ $\pi /\left(2 \omega_{c}\right)$. This means that the maximum instantaneous frequency excursion contained in the spectrum will be 
$\omega_{m}$. Therefore, as the amplitude or frequency of the modulation increases, the spectrum will become broader. Hence, by means of a simple physical argument, we can make the same observation about the spectral width of a phase-modulated signal as that deduced from Eq. (4) and the plots of Bessel functions, and we are also able to obtain a rough estimate of such width. Now we discuss the origin of the oscillations that can be observed in the spectrum.

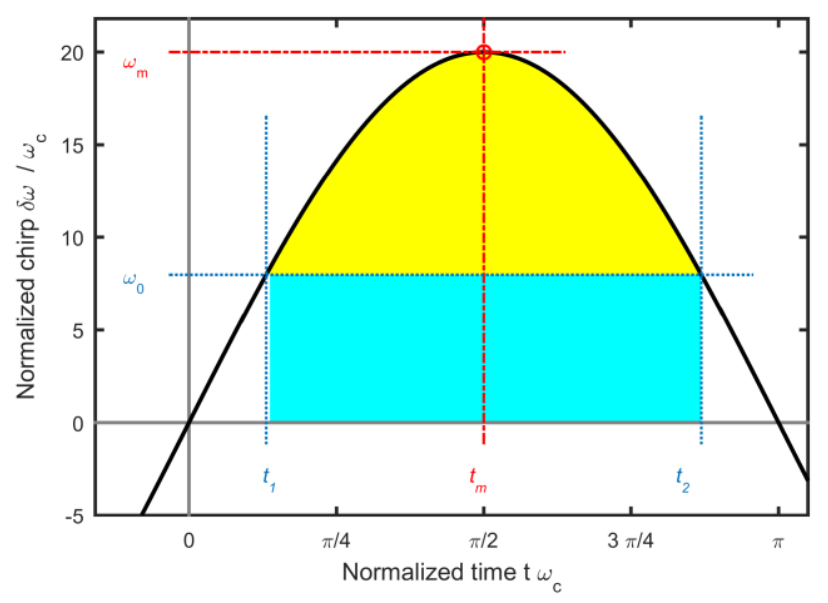

Figure 4 - Temporal variation of the chirp of a continuous wave experiencing a sinusoidal phase modulation of amplitude $A=20 \mathrm{rad}$, as viewed within a half-period of oscillation. The position of the maximum of the function and the solutions of the equation $\delta \omega=\omega_{0}$ are indicated with red and blue lines, respectively. The cyan and yellow regions represent the phase difference $\Delta \varphi_{D}$ and the opposite of the total phase offset $\Delta \varphi_{T}$, respectively.

\subsection{Determination of the instants involved in the interference process}

We can deduce from the chirp function of Eq. (5) that a given instantaneous frequency $\omega_{0}$ between 0 and $\omega_{m}$ can be observed at two different instants, $t_{1}$ and $t_{2}$, within a half-period. These instants are the two roots of the equation $\delta \omega(t)=\omega_{0}$ over the time interval being considered:

$$
\left\{\begin{array}{l}
t_{1}=\frac{1}{\omega_{c}} \arcsin \left(\frac{\omega_{0}}{\omega_{m}}\right) \\
t_{2}=\frac{\pi}{\omega_{c}}-t_{1}
\end{array}\right.
$$

Therefore, the wave with frequency $\omega_{0}$ will interfere with its replica delayed by the time

$$
\Delta t=t_{2}-t_{1}=\frac{\pi}{\omega_{c}}-2 t_{1}=\frac{1}{\omega_{c}}\left(\pi-2 \arcsin \left(\frac{\omega_{0}}{\omega_{m}}\right)\right)
$$

in a similar manner to when a beam is combined with a delayed replica of itself in double-path experiments such as the Michelson or Mach-Zehnder interferometers [13, 14]. Another example of the occurrence of twowave interference is when non-monochromatic light experiences a time delay passing through an anisotropic wave plate. Because of the time delay $\Delta t$, the two waves will create constructive or destructive interference, thus engendering a modulation of the intensity of the spectrum, which will map out the total difference in phase between the two waves.

\subsection{Calculation of the different phase contributions}

The total phase difference $\Delta \varphi_{T}$ between the two instants $t_{1}$ and $t_{2}$ is the result of two contributions. The first contribution $\Delta \varphi_{D}$ is due to the time delay $\Delta t$ : 


$$
\Delta \varphi_{D}=\omega_{0} \Delta t=\frac{\omega_{0}}{\omega_{c}}\left(\pi-2 \arcsin \left(\frac{\omega_{0}}{\omega_{m}}\right)\right)
$$

This quantity can be represented graphically in Fig. 4 as the area of the cyan rectangle of width $\omega_{0}$ and length $\Delta t$. One may readily see from Eq. (8) and Fig. 5 (solid red curve) or infer from Fig. 4 that $\Delta \varphi_{D}$ is a nonmonotonic function of the instantaneous frequency $\omega_{0}$, and $\Delta \varphi_{D}=0$ for both $\omega_{0}=0$ and $\omega_{0}=\omega_{m}$.

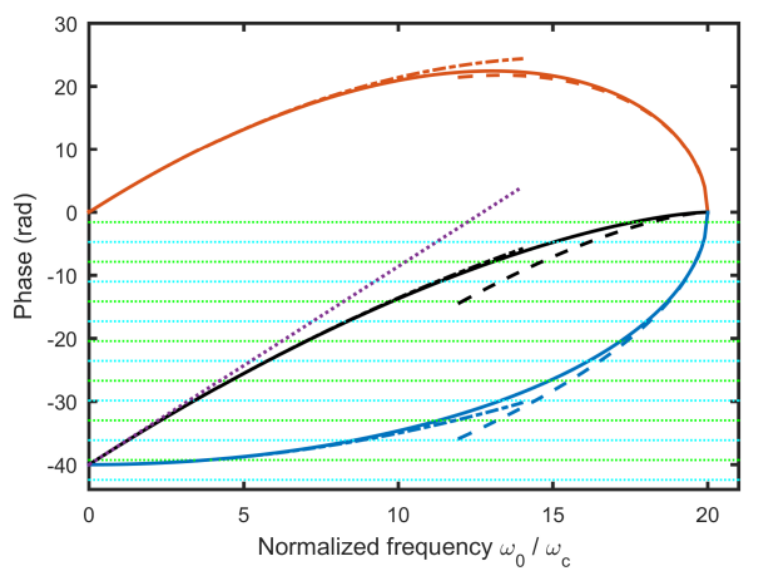

Figure 5 - Variation of the different phase contributions with the instantaneous frequency $\omega_{0}$ for a continuous wave experiencing a sinusoidal phase modulation of amplitude $A=20 \mathrm{rad}$. The phase differences $\Delta \varphi_{D}, \Delta \varphi_{E}$ and $\Delta \varphi_{\mathrm{T}}$ as calculated from Eqs. (8), (10) and (12) are represented by red, blue and black solid curves, respectively. Their low-frequency approximations based on Eq. (21), (22) and (23), and high-frequency approximations based on Eqs. (34), (35) and (36) are represented by dash-dotted and dashed curves, respectively, of the corresponding color. The linear approximation of the total phase difference at low frequencies [Eq. (26)] is plotted with a dotted purple line. The green and cyan horizontal lines represent the values of the total phase difference causing constructive and destructive interference, respectively [Eqs. (17) and (16)].

The second contribution $\Delta \varphi_{E}$ to the total phase difference arises from the phase modulation itself:

$$
\Delta \varphi_{E}=\varphi\left(t_{2}\right)-\varphi\left(t_{1}\right)=A\left[\cos \left(\omega_{c} t_{2}\right)-\cos \left(\omega_{c} t_{1}\right)\right]
$$

Using the trigonometric identity for the difference of two cosines and noticing that

$$
\sin \left(\frac{\omega_{c}}{2}\left(t_{2}+t_{1}\right)\right)=\sin \left(\frac{\omega_{c}}{2} \frac{\pi}{\omega_{c}}\right)=1
$$

we can cast Eq. (9) into

$$
\begin{aligned}
\Delta \varphi_{E} & =-2 A \sin \left(\frac{\omega_{c}}{2}\left(t_{2}+t_{1}\right)\right) \sin \left(\frac{\omega_{c}}{2}\left(t_{2}-t_{1}\right)\right) \\
& =-2 A \sin \left(\frac{\omega_{c}}{2} \Delta t\right)=-2 A \sin \left(\frac{\pi}{2}-\arcsin \left(\frac{\omega_{0}}{\omega_{m}}\right)\right)=-2 A \cos \left(\arcsin \left(\frac{\omega_{0}}{\omega_{m}}\right)\right)
\end{aligned}
$$

Applying the Pythagorean theorem to the right triangle of hypotenuse $\omega_{m}$ and one side $\omega_{0}$, we obtain

$$
\cos \left(\arcsin \left(\frac{\omega_{0}}{\omega_{m}}\right)\right)=\frac{\sqrt{\omega_{m}^{2}-\omega_{0}^{2}}}{\omega_{m}}=\sqrt{1-\left(\frac{\omega_{0}}{\omega_{m}}\right)^{2}}
$$

and

$$
\Delta \varphi_{E}=-2 A \sqrt{1-\left(\frac{\omega_{0}}{\omega_{m}}\right)^{2}}
$$


Note that Eq. (10) can be written in the form

$$
\left(\frac{\Delta \varphi_{E}}{2 A}\right)^{2}+\left(\frac{\omega_{0}}{\omega_{m}}\right)^{2}=1
$$

which is the equation of an ellipse with the semi-axes $2 A$ and $\omega_{m}$. Therefore, $\Delta \varphi_{E}\left(\omega_{0}\right)$ is a quarter of an ellipse as can be seen in Fig. 5 (solid blue curve).

This phase contribution can also be calculated as the integral of the opposite of the chirp function, $-\delta \omega(t)$, over the interval $\left[t_{1}, t_{2}\right]$, thereby represented as the opposite of the area under the curve $\delta \omega(t)$ between $t=t_{1}$ and $t=$ $t_{2}$ in Fig. 4. It is apparent that $\Delta \varphi_{E}$ is a negative and monotonically increasing function of $\omega_{0}$ that has the maximum negative value of $-2 A$ at $\omega_{0}=0$ and is zero when $\omega_{0}=\omega_{m}$ (i.e. $t_{l}=t_{2}$ ).

The total phase difference between $t_{1}$ and $t_{2}$ is therefore:

$$
\Delta \varphi_{T}\left(\omega_{0}\right)=\Delta \varphi_{D}+\Delta \varphi_{E}=\frac{\omega_{0}}{\omega_{c}}\left(\pi-2 \arcsin \left(\frac{\omega_{0}}{\omega_{m}}\right)\right)-2 A \sqrt{1-\left(\frac{\omega_{0}}{\omega_{m}}\right)^{2}}
$$

It can be geometrically interpreted as the opposite of the area of the region in Fig. 4 that is bounded by the graph of $\delta \omega$ and the horizontal line $\delta \omega=\omega_{0}$, and it is a negative and monotonically increasing function of $\omega_{0}$ with the same extrema as $\Delta \varphi_{E}$ (Fig. 5, solid black curve).

\subsection{Derivation of the intensity in the interference pattern}

With the knowledge of the instants $t_{1}$ and $t_{2}$ and the total phase difference $\Delta \varphi_{T}$ between them, we can calculate the distribution of intensity in the pattern associated with the interference between these two times. The two electric fields superposed at frequency $\omega_{0}$ are

$$
\left\{\begin{array}{l}
\psi_{1}=\psi_{0} e^{i \varphi\left(t_{1}\right)} e^{i \omega_{0} t_{1}} \\
\psi_{2}=\psi_{0} e^{i \varphi\left(t_{2}\right)} e^{i \omega_{0} t_{2}}
\end{array}\right.
$$

Thus, the resultant wave at $\omega_{0}$ is

$$
\begin{gathered}
\psi_{T}\left(\omega_{0}\right)=\psi_{1}+\psi_{2}=\psi_{0} e^{i \varphi\left(t_{1}\right)} e^{i \omega_{0} t_{1}}+\psi_{0} e^{i \varphi\left(t_{2}\right)} e^{i \omega_{0} t_{2}} \\
\psi_{T}\left(\omega_{0}\right)=\psi_{0} e^{i \varphi\left(t_{1}\right)} e^{i \omega_{0} t_{1}}\left(1+e^{i\left(\varphi\left(t_{2}\right)-\varphi\left(t_{1}\right)\right)} e^{i \omega_{0}\left(t_{2}-t_{1}\right)}\right)=\psi_{0} e^{i \varphi\left(t_{1}\right)} e^{i \omega_{0} t_{1}}\left(1+e^{i \Delta \varphi_{T}}\right)
\end{gathered}
$$

The intensity of this wave is proportional to the modulus squared:

$$
\begin{gathered}
I\left(\omega_{0}\right) \propto \psi_{T} \psi_{T}^{*}=\left|\psi_{0}\right|^{2}\left(1+e^{i \Delta \varphi_{T}}\right)\left(1+e^{-i \Delta \varphi_{T}}\right) \\
I\left(\omega_{0}\right) \propto 1+\cos \left(\Delta \varphi_{T}\right)
\end{gathered}
$$

For reasons that go beyond the scope of this paper, we need to account for an additional $\pi / 2$ phase shift in the expression above. Hence, we arrive at our final result:

$$
I\left(\omega_{0}\right) \propto 1-\sin \left(\Delta \varphi_{T}\right)
$$

Therefore, the total intensity oscillates with the phase difference between the two waves. Note that as $\Delta \varphi_{T}$ is not a linear function of $\omega_{0}$, these oscillations are not strictly periodic and the extent of an oscillation varies with $\omega_{0}$. Figure 6 compares the spectral intensity distribution as calculated from Eq. (15) with the spectrum $S_{n}$ obtained from Eq. (4), and apparently indicates a good agreement between the results of our qualitative approach and those of the rigorous Fourier-analysis approach. However, we can also note some differences: $I\left(\omega_{0}\right)$ is defined on the frequency interval $\left[0, \omega_{m}\right]$ (and the symmetric interval about 0 ) and, thus, it cannot account for components in the spectrum above $\omega_{m}$. Moreover, the intensity of the outermost peak of $S_{n}$ is higher than the intensity calculated from Eq. (15) at that frequency. This can be qualitatively understood by considering that the probability density function of a sinusoidal wave has peaks at the extrema of the sinusoid. 


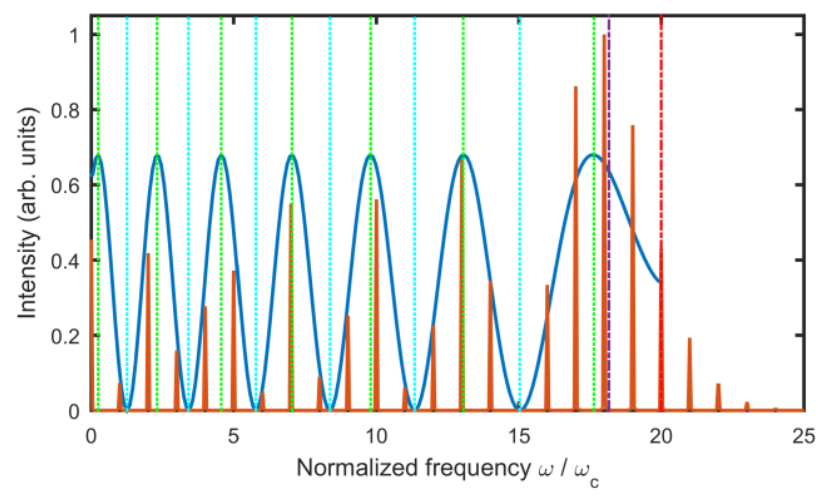

Figure 6 - Spectral intensity distribution as calculated from Eq. (15) (blue solid curve) compared to the spectrum $S_{n}$ obtained from Eq. (4) (red solid lines). The green and cyan dotted lines represent the frequencies at which constructive and destructive interference occurs, as predicted by Eqs. (17) and (16), respectively. The red and purple dash-dotted lines represent the maximum frequency $\omega_{m}$ and the position $\omega_{M}$ of the outermost spectral peak as predicted by Eq. (37).

\subsection{Positions of the extrema of the spectral intensity}

Equation (15) indicates that there is destructive interference causing intensity minima at frequencies for which

$$
\Delta \varphi_{T}=\left(2 m+\frac{1}{2}\right) \pi, \quad m=-1,-2,-3, \ldots
$$

Intensity maxima or constructive interference occur when

$$
\Delta \varphi_{T}=\left(2 m-\frac{1}{2}\right) \pi, \quad m=0,-1,-2,-3, \ldots
$$

In particular, the position $\omega_{M}$ of the outermost peak of intensity corresponds to $m=0$ in Eq. (17) :

$$
\Delta \varphi_{T}\left(\omega_{M}\right)=-\frac{\pi}{2}
$$

From Eq. (17), one may also derive a convenient relationship between the number of peaks $k$ contained in the spectrum and the value of $A[15]$ :

$$
A \simeq \frac{\pi}{2}\left(k-\frac{1}{2}\right)
$$

\subsection{Calculation of the spectrum}

Equation (15) provides an approximate shape for the envelope of the spectrum of the phase-modulated signal. But it does not reproduce the comb structure of the actual spectrum as it has been derived by considering the spectral interference that occurs over a single period of oscillation of the phase modulation. If we take into account the spectral interferences occurring over each oscillatory period, we will obtain a series of spikes of infinitesimal width (Dirac delta functions), similarly to a Fabry-Perot interferometer where the superposition of a large number of offset beams causes an interference pattern with much sharper fringes than those obtained with two-beam interference $[16,17]$. These spikes will be equally spaced by $\omega_{c}$.

This discrete nature of the spectrum can be accounted for in our calculation by computing the total phase difference between two interfering waves at sequential integer values of $\omega_{0} / \omega_{c}$ :

$$
\Delta \varphi_{T}(n)=n\left(\pi-2 \arcsin \left(\frac{n}{A}\right)\right)-2 \sqrt{A^{2}-n^{2}}, \quad n=0, \pm 1, \pm 2, \pm 3, \ldots, \quad|n| \leq A
$$

The resulting spectrum, $I_{n}$, is plotted in Fig. 7 as a function of the amplitude of the modulation $A$, showing good qualitative agreement with the results of the Fourier-analysis calculation. 


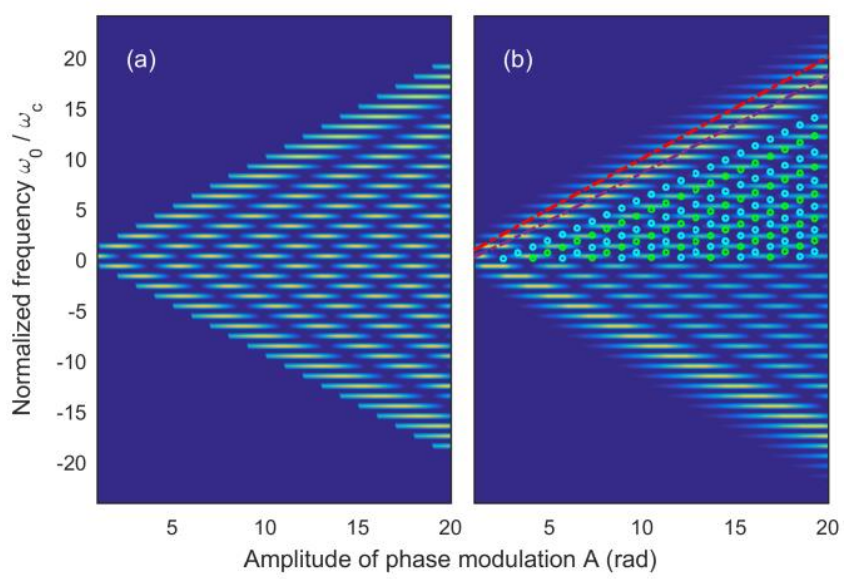

Figure 7 - Evolution of the spectrum with the amplitude of the phase modulation. The spectrum $I_{n}$ calculated based on Eq. (20) (subplot a) is compared with the spectrum $S_{n}$ obtained from Eq. (4) (subplot b). The green and cyan circles represent the frequencies at which constructive and destructive interference occurs, as predicted by Eqs. (25) and (24), respectively. The red and purple dashdotted lines represent the maximum frequency $\omega_{m}$ and the position $\omega_{M}$ of the outermost spectral peak as predicted by Eq. (37).

\section{Further simplifications}

The interference model developed in the previous section can provide an understanding of the origin of the oscillatory structure of a phase-modulated signal's spectrum. Now we will show that very simple approximate formulae can be obtained within this model that are able to capture the main features of the central part of the spectrum and the spectrum's edges.

\subsection{Prediction of the behaviour at low frequencies based on Taylor expansion}

First we consider the low-frequency components of the spectrum, i.e. we suppose the frequency $\omega_{0}$ is much smaller than $\omega_{m}$. In this case $\arcsin \left(\omega_{0} / \omega_{m}\right)$ is very nearly equal to $\omega_{0} / \omega_{m}$, and thus the phase contribution $\Delta \varphi_{D}$ of Eq. (8) can be approximated by (see Fig. 5, red dashed line):

$$
\Delta \varphi_{D} \simeq \frac{\omega_{0}}{\omega_{c}}\left(\pi-2 \frac{\omega_{0}}{\omega_{m}}\right) \simeq \pi \frac{\omega_{0}}{\omega_{c}}-2 A\left(\frac{\omega_{0}}{\omega_{m}}\right)^{2}
$$

Using the binomial expansion $(1+\varepsilon)^{p} \simeq 1+p \varepsilon+\ldots$ with $p=1 / 2$ and $\varepsilon=-\left(\omega_{0} / \omega_{m}\right)^{2}$, the phase contribution $\Delta \varphi_{E}$ of Eq. (10) can be approximated by (see Fig. 5, blue dashed line)

$$
\Delta \varphi_{E} \simeq-2 A\left(1-\frac{1}{2}\left(\frac{\omega_{0}}{\omega_{m}}\right)^{2}\right)=-2 A+A\left(\frac{\omega_{0}}{\omega_{m}}\right)^{2}
$$

We therefore obtain the following expansion for the total phase difference $\Delta \varphi_{T}$ of Eq. (12) about the central frequency:

$$
\Delta \varphi_{T} \simeq-2 A+\pi \frac{\omega_{0}}{\omega_{c}}-2 A\left(\frac{\omega_{0}}{\omega_{m}}\right)^{2}+A\left(\frac{\omega_{0}}{\omega_{m}}\right)^{2}=A\left(-2+\pi \frac{\omega_{0}}{\omega_{m}}-\left(\frac{\omega_{0}}{\omega_{m}}\right)^{2}\right)
$$

As we can see in Fig. 5 (dash-dotted black curve), this expansion provides a rather good approximation to the actual function over a wide range of frequencies. Equations (16) and (17) giving the positions of the intensity extrema in the spectral modulation pattern can now be readily solved, hence obtaining

$$
\omega_{0} \simeq \omega_{m}\left[\frac{\pi}{2}-\sqrt{\frac{\pi^{2}}{4}-2-\left(2 m+\frac{1}{2}\right) \frac{\pi}{A}}\right], \quad m=0,-1,-2,-3, \ldots
$$

for the intensity minima, and 


$$
\omega_{0} \simeq \omega_{m}\left[\frac{\pi}{2}-\sqrt{\frac{\pi^{2}}{4}-2-\left(2 m-\frac{1}{2}\right) \frac{\pi}{A}}\right], \quad m=0,-1,-2,-3, \ldots
$$

for the intensity maxima. The predictions from Eq. (24) and Eq. (25) show to be fairly accurate [cyan and green vertical lines, respectively, in Fig. 8(a)].

If we approximate $\Delta \varphi_{T}$ with the first term of its Taylor series (purple dotted line in Fig. 5),

$$
\Delta \varphi_{T} \simeq-2 A+\pi \frac{\omega_{0}}{\omega_{c}}
$$

then the resulting spectrum's envelope takes the form of a sinusoidally oscillating function in the central region:

$$
I\left(\omega_{0}\right) \propto 1+\sin \left(2 A-\pi \frac{\omega_{0}}{\omega_{c}}\right)
$$

and the amplitude of the $n^{\text {th }}$ spectral component becomes:

$$
I_{n} \propto 1+\sin (2 A-n \pi)=1+(-1)^{n} \sin (2 A)
$$

Equation (28) indicates that the difference in intensity $\Delta I$ between two successive spectral components in the central part of the spectrum is $2 \sin (2 A)$. It is interesting to notice that particular values of $A$ will lead to interesting spectral patterns. For instance, when $A=m \pi / 2$ with $m$ integer, $\Delta I=0$, so all the components at the centre of the spectrum will have the same intensity level, in accordance with the characteristic of the spectrum obtained from Eq. (4) for such values of $A$ [Fig. 8(b1)]. Further, when $A=(2 m+1) \pi / 4$, one every two components vanishes, which again reproduces the trend observable in $S_{n}$ [Fig. 8(b2)].

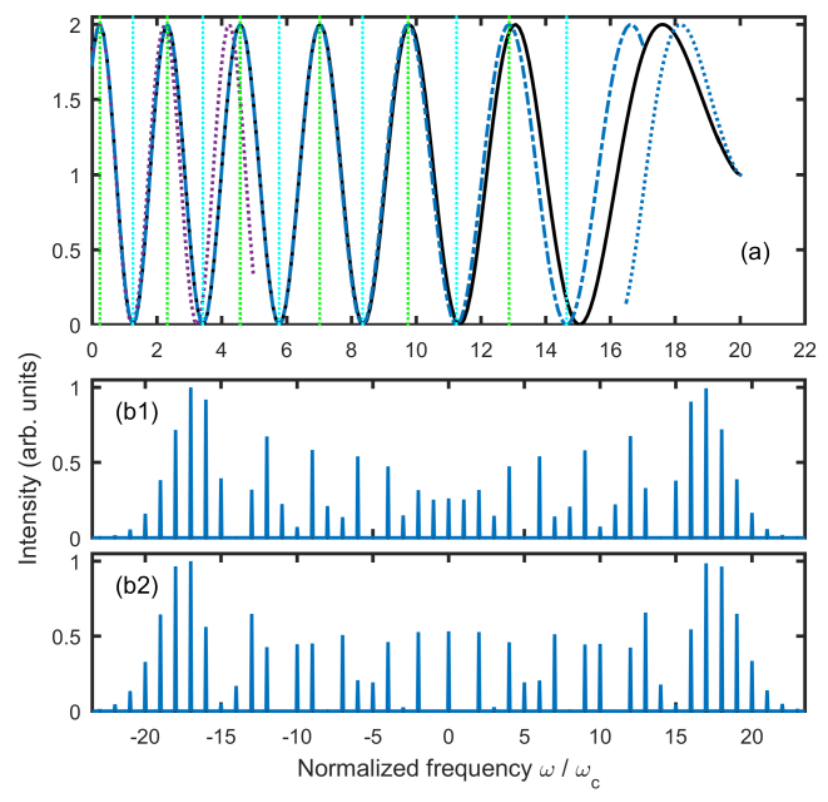

Figure 8- (a) Comparison of various approximations to the envelope of the spectrum of a continuous wave experiencing a phase modulation of amplitude $A=20 \mathrm{rad}$. The solid black curve represents the spectrum's envelope as calculated from Eqs. (12) and (15). The dash-dotted blue and dotted purple curves represent the low-frequency approximation based on the Taylor expansion of the phase difference $\Delta \varphi_{T}$ [Eq. (23)] and the low-frequency approximation given by Eq. (27) and based on the linear approximation of $\Delta \varphi_{T}$ [Eq. (26)], respectively. The dotted blue curve represents the high-frequency approximation based on Eq. (36). The green and cyan dotted lines represent the frequencies at which constructive and destructive interference occurs, as predicted by Eqs. (25) and (24), respectively. (b) Spectra $S_{n}$ obtained from Eq. (4) for $A=6 \pi$ (subplot 1) and $A=12.5 \pi / 2$ (subplot 2). 
It can also be interesting to understand the oscillations of the amplitudes of the spectral components $I_{n}$ as a function of the modulating phase's amplitude $A$. We consider here the behavior at large $A$ (which falls into the present limit of low frequencies). Hence, using the double-angle formula for cosine:

$$
I_{n}(A) \propto 1+\sin (2 A-n \pi)=1+\cos \left(2 A-n \pi-\frac{\pi}{2}\right)=2 \cos ^{2}\left(A-n \frac{\pi}{2}-\frac{\pi}{4}\right)
$$

Further, we observe that the maximum frequency contained in the spectrum $\omega_{m}$ increases with increasing values of $A$. As the energy content of the spectrum is not modified by a modulation of the phase the area under the curve of the spectrum is conserved, hence an increase in the bandwidth should be accompanied by a reduction of the amplitude by the same factor. Therefore, we can introduce a $1 / A$ dependence of the spectral envelope $I\left(\omega_{0}\right)$, leading to

$$
I_{n}(A) \propto \frac{2}{A} \cos ^{2}\left(A-n \frac{\pi}{2}-\frac{\pi}{4}\right)
$$

Now if we notice that the Bessel functions of the first kind have the following asymptotic form for large arguments [3,4] (Fig. 2, red dotted curves)

$$
J_{n}(x) \sim \sqrt{\frac{2}{\pi x}} \cos \left(x-\frac{\pi}{2} n-\frac{\pi}{4}\right)
$$

we can recognize that the frequency spectrum of the signal calculated based on Eq. (4) has the same asymptotic behavior for large $A$ as that derived here without relying on any knowledge of Bessel functions:

$$
S_{n} \propto J_{n}^{2}(A) \sim \frac{2}{\pi A} \cos ^{2}\left(A-\frac{\pi}{2} n-\frac{\pi}{4}\right)
$$

\subsection{Prediction of the behaviour at low frequencies based on geometric interpretation}

Another way of gaining insight into the behavior of the total phase difference $\Delta \varphi_{T}$ at low frequencies is to use the graphical interpretation of the function given in Fig. 4 (see also Fig. 9). Indeed, the area of the region bounded by the graph of the chirp function $\delta \omega$ and the horizontal line $\delta \omega=\omega_{0}$ can be calculated as the difference between the area $a_{1}$ under the curve $\delta \omega$ over a half-period of oscillation:

$$
a_{1}=\int_{0}^{\pi / \omega_{C}} A \omega_{C} \sin \left(\omega_{C} t\right) d t=2 A
$$

and the area $a_{2}$ of the region that, at low frequencies, has the shape of an isosceles trapezoid with the bottom basis of length $\pi / \omega_{C}$ and a height of $\omega_{0}$ (Fig. 9, pink region). Since the slope of $\delta \omega$ at the origin is $A \omega_{C}^{2}$, we can easily calculate the length of the top basis of the trapezoid by $\pi / \omega_{C}-2 \omega_{0} /\left(A \omega_{C}^{2}\right)$. Hence, we obtain

$$
a_{2}=\frac{\omega_{0}}{2}\left(\frac{\pi}{\omega_{C}}+\frac{\pi}{\omega_{C}}-2 \frac{\omega_{0}}{A \omega_{C}^{2}}\right)=\frac{\pi \omega_{0}}{\omega_{C}}-\frac{\omega_{0}^{2}}{A \omega_{C}^{2}}
$$

and

$$
\Delta \varphi_{T}=-\left(a_{1}-a_{2}\right)=-2 A+\pi \frac{\omega_{0}}{\omega_{C}}+\frac{\omega_{0}^{2}}{A \omega_{C}^{2}}=A\left(-2+\pi \frac{\omega_{0}}{\omega_{m}}-\left(\frac{\omega_{0}}{\omega_{m}}\right)^{2}\right)
$$

Therefore, we have attained the same expression for $\Delta \varphi_{T}$ as given by Eq. (23) without using any Taylor-series expansion. 


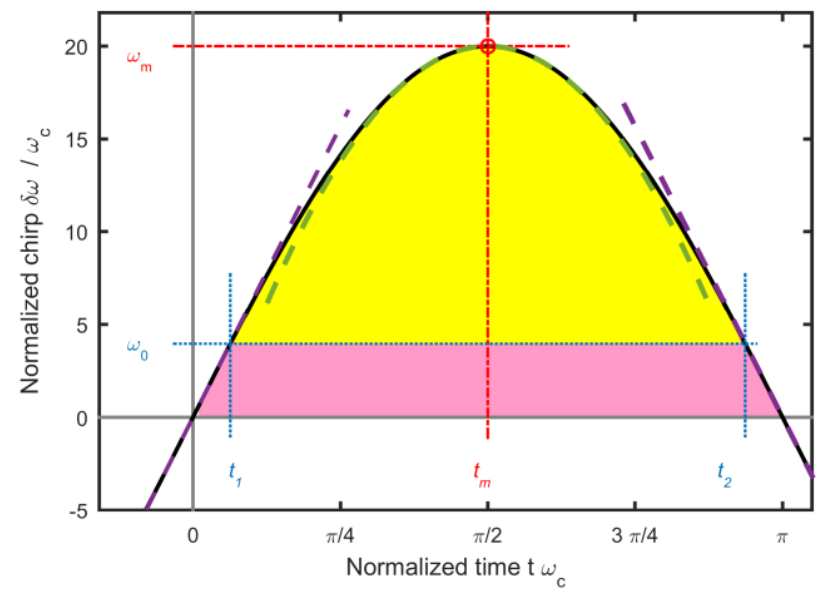

Figure 9 Geometric interpretation of the approximate calculation of the total phase difference $\Delta \varphi_{T}$ at low frequencies: the yellow region bounded by the graph of the chirp function $\delta \omega$ and the horizontal line $\delta \omega=\omega_{0}$ is the opposite of $\Delta \varphi_{T}$. The purple dashed lines represent the linear approximation to the chirp function near $t=0$ and $t=\pi / \omega \mathrm{c}$. Also shown is the inverted parabola approximating the chirp function at high frequencies (green dashed curve).

\subsection{Prediction of the behaviour at high frequencies}

In the previous section, we have gained insights into the characteristics of the central part of the optical spectrum and its behavior for large amplitudes of the phase modulation. We now focus on the edges of the spectrum, where $\omega_{0}$ is close to $\omega_{m}$. In the vicinity of $x=\pi / 2, \sin (x)$ can be approximated by an inverted parabola (Fig. 9, green dashed curve):

$$
\sin \left(\frac{\pi}{2}+\varepsilon\right) \simeq 1-\frac{\varepsilon^{2}}{2}
$$

Accordingly, the equation $\delta \omega(t)=\omega_{0}$ takes the form

$$
1-\frac{1}{2}\left(\omega_{C} t-\frac{\pi}{2}\right)^{2} \simeq \frac{\omega_{0}}{\omega_{m}}
$$

and has the roots

$$
\omega_{C} \quad t_{1,2}=\frac{\pi}{2} \pm \sqrt{2\left(1-\frac{\omega_{0}}{\omega_{m}}\right)}
$$

Plugging the resulting delay $\Delta t=t_{1}-t_{2}$ into the expression of the phase difference $\Delta \varphi_{D}$, we obtain (Fig. 5, red dashed curve)

$$
\Delta \varphi_{D}=2 \frac{\omega_{0}}{\omega_{C}} \sqrt{2\left(1-\frac{\omega_{0}}{\omega_{m}}\right)}
$$

When $\omega_{0}$ tends to $\omega_{m}$, the phase difference $\Delta \varphi_{E}$ between $t_{1}$ and $t_{2}$ due to the external phase modulation can be approximated by (Fig. 5, blue dashed curve)

$$
\Delta \varphi_{E}=-2 A \sqrt{1-\left(\frac{\omega_{0}}{\omega_{m}}\right)^{2}}=-2 A \sqrt{\left(1-\frac{\omega_{0}}{\omega_{m}}\right)\left(1+\frac{\omega_{0}}{\omega_{m}}\right)} \simeq-2 A \sqrt{2\left(1-\frac{\omega_{0}}{\omega_{m}}\right)}
$$

Therefore, the total phase difference becomes

$$
\Delta \varphi_{T}=2 \frac{\omega_{0}}{\omega_{C}} \sqrt{2\left(1-\frac{\omega_{0}}{\omega_{m}}\right)}-2 A \sqrt{2\left(1-\frac{\omega_{0}}{\omega_{m}}\right)}=2^{3 / 2} \sqrt{\left(1-\frac{\omega_{0}}{\omega_{m}}\right)}\left(\frac{\omega_{0}}{\omega_{C}}-A\right)
$$


or (Fig. 5, black dashed curve)

$$
\Delta \varphi_{T}=-2^{\frac{3}{2}} A\left(1-\frac{\omega_{0}}{\omega_{m}}\right)^{\frac{3}{2}}
$$

It is therefore possible to obtain a closed formula for the location $\omega_{M}$ of the maximum of the outermost oscillation of the spectrum [calculated based on Eq. (18)]:

$$
\omega_{M}=\omega_{m}\left[1-\frac{1}{2}\left(\frac{\pi}{2 A}\right)^{2 / 3}\right]
$$

The value of $\omega_{M}$ predicted by Eq. (37) is plotted on Fig. 6 and 7, where we can see that it provides a rather good estimate of the position of the external spectral peak.

\section{Conclusion}

We have described the effects of a sinusoidal phase modulation on the spectrum of an optical continuous wave. Rather than relying on the standard Fourier-analysis approach involving special mathematical functions that students may not always master, we have regarded the process as an interference effect. By calculating the phase difference between the two times at which the instantaneous frequency reaches each value, we have been able to obtain the spectral envelope of the signal and to understand the reason for the oscillatory character of the spectrum. This reason is essentially that the instantaneous frequency undergoes strong excursions, so that in general there are contributions from two different times to the Fourier integral for a given frequency component. Depending on the exact frequency, these contributions may constructively add up or cancel each other. We note that this comb structure of the spectrum is also typical of other periodic interferometric systems such as a Fabry-Perot resonator. Without assuming any knowledge of the general trends of the Bessel functions, our model can correctly predict the behaviour of the spectrum for large amplitudes of the phase modulation and provide an estimate for the positions of the extrema of the spectrum.

Our proposed approach can be extended to other physical processes such as the self-phase modulation that occurs in nonlinear optical media [18], especially in fibers [19], by which an intense optical pulse induces through the third-order nonlinearity of the medium a modulation of its phase that is proportional to its own intensity profile. The substantial spectrum broadening that may ensue from this finds various applications such as in multi-wavelength optical sources [20]. It is worthwhile to mention that the interference model described in this paper relies on the same idea of the method of stationary phase, i.e., the cancellation of oscillatory contributions with rapidly varying phase. This powerful analytic tool for obtaining the leading asymptotic behaviour of generalised Fourier integrals where the phase has distinct stationary points [21] is based on the assumption that there are no significant contributions to the Fourier integral from fast varying phase terms with frequency, but only from a small interval surrounding the stationary points of the phase. The stationary phase approximation, however, is no longer valid in our case when the instantaneous frequency is close to its maximum excursion: in this case indeed, the deviations of our model from the results of the Fourier-analysis approach are typically larger.

Our work confirms once more that the field of optics is very well suited for acquiring an appreciation of the Fourier transform theory [22].

\section{Acknowledgement}

The authors would like to acknowledge support by the Institut Universitaire de France and the Agence Nationale de la Recherche (Labex Action ANR-11-LABX-01-01). 


\section{References}

[1] Al-Amri MD, El-Gomati M, Zubairy MS 2016 Optics in Our Time: Springer.

[2] Fourier J 1822 Theorie analytique de la chaleur: Firmin Didot, père et fils.

[3] Weisstein EW. Bessel Function of the First Kind: From MathWorld--A Wolfram Web Resource; [Available from: http://mathworld.wolfram.com/BesselFunctionoftheFirstKind.html.

[4] Wikipedia contributors. Bessel function: Wikipedia, The Free Encyclopedia.; [Available from: https://en.wikipedia.org/w/index.php?title=Bessel_function.

[5] Hecht E 2002 Optics, 4th. San Francisco: International edition, Addison-Wesley.

[6] Wikipedia contributors. Vibrations of a circular membrane: Wikipedia, The Free Encyclopedia.; [Available from: https://en.wikipedia.org/wiki/Vibrations of a circular membrane.

[7] Hecht E Physics : Calculus.

[8] Crawford F Waves : Berkeley Physics Course Volume 3.

[9] Godil AA, Auld BA, Bloom DM 1994 Picosecond time-lenses IEEE J Quantum Electron 30 827-37.

[10] Varlot B, Wabnitz S, Fatome J, Millot G, Finot C 2013 Experimental generation of optical flaticon pulses Opt Lett 38 3899-902.

[11] Komukai T, Yamamoto Y, Kawanishi S 2005 Optical pulse generator using phase modulator and linearly chirped fiber Bragg gratings IEEE Photon Technol Lett 17 1746-8.

[12] Nuno J, Finot C, Fatome J 2017 Linear Sampling and Magnification Technique Based on Phase Modulators and Dispersive Elements: the Temporal Lenticular Lens Opt Fiber Technol 36 125-9.

[13] Nachman P 1995 Mach-Zehnder interferometer as an instructional tool American Journal of Physics 63 $39-43$.

[14] Cavalcanti CJH, Ostermann F, Lima NW, Netto JS 2017 Software-aided discussion about classical picture of Mach-Zehnder interferometer Eur J Phys 38065703.

[15] Cubeddu R, Polloni R, Sacchi CA, Svelto O 1970 Self-Phase Modulation and "Rocking" of Molecules in Trapped Filaments of Light with Picosecond Pulses Physical Review A 2 1955-63.

[16] Perot A, Fabry C 1899 On the Application of Interference Phenomena to the Solution of Various Problems of Spectroscopy and Metrology Astrophysical Journal 987.

[17] Sánchez-Soto LL, Monzón JJ, Leuchs G 2016 The many facets of the Fabry-Perot Eur J Phys 37064001.

[18] Shimizu F 1967 Frequency Broadening in Liquids by a Short Light Pulse Phys Rev Lett 19 1097-100.

[19] Stolen RH, Lin C 1978 Self-phase modulation in silica optical fibers Phys Rev A 17 1448-53.

[20] Parmigiani F, Finot C, Mukasa K, Ibsen M, Roelens MAF, Petropoulos P, et al. 2006 Ultra-flat SPMbroadened spectra in a highly nonlinear fiber using parabolic pulses formed in a fiber Bragg grating $O p t$ Express 14 7617-22.

[21] Copson JET 1965 Asymptotic Expansions: Cambridge University Press. 
[22] Ceffa NG, Collini M, D’Alfonso L, Chirico G 2016 Hands-on Fourier analysis by means of far-field diffraction Eur J Phys 37065701. 